\title{
RÉPARTITION DES STRUCTURES ARGYROPHILES CHEZ DEUX CERCAIRES DE STRIGÉIDES COTYLURUS BREVIS DUBOIS ET RAUSGH, 1950 ET APATEMON (A.) MINOR YAMAGUTI, 1933.
}

\author{
J. RICHARD*
}

\begin{abstract}
RÉSUMÉ. Deux furcocercaires respectivement Cotylurus brevis Dubois et Rausch, 1950 de Segmentina nitida et Apatemon (A.) minor Yamaguti, 1933 de Gyraulus acronicus sont étudiées et déterminées en fonction de leur morphologie.

La distribution des papilles ciliaires est étudiée après imprégnation par une solution de nitrate d'argent et comparée à celle des cercaires connues dans le même genre. Cotylurus brevis est comparée à Cotylurus lutzi Basch, 1969 et Cotylurus sp. Kruger, 1978. Apatemon (A.) minor est comparée à Apatemon sp. Kruger, 1978, Apatemon graciliformis Szidat, 1928 et Cercaria 1 Richard, 1971. Les cercaires appartenant aux genres Cotylurus et Apatemon sont aisément identifiables d'après le nombre et la distribution des papilles acétabulaires : 4 SI +6 SII dans le genre Cotylurus, $3 \mathrm{SI}+3 \mathrm{SII}$ dans le genre Apatemon. Par extension ces caractéristiques permettraient d'identifier une cercaire du genre Diplostomum puisque toutes les cercaires étudiées dans ce genre ont $3 \mathrm{SI}+$ 6 SII.

Apatemon graciliformis est une exception. Or nous avons remarqué des variations des papilles acétabulaires chez Cercaria 1. On pourrait admettre que $A$. graciliformis correspond à un stade évolutif particulier, mais en l'absence de données sur les variations du nombre et de la distribution des papilles acétabulaires chez les autres espèces, nous ne pouvons écarter l'hypothèse de son appartenance à une sous genre ou même à un genre différent.
\end{abstract}

\section{Distribution of argentophilic structures in two Strigeid cercariae, Cotylurus brevis Dubois et Rausch, 1950 and Apatemon (A.) minor Yamaguti, 1933.}

SUMMARY. Two furcocercariae respectively Colylurus brevis Dubois et Rausch, 1950 from Segmentina nitida and Apatemon (A.) minor Yamaguti, 1933 from Gyraulus acronicus were studied and identified morphologically.

Ciliary papillae distribution was studied after staining with silver nitrate solution and compared with that of cercariae known in the same genus. Colylurus brevis was compared to Cotylurus lutzi Basch, 1969 and Cotylurus sp. Kruger, 1978. Apatemon (A.) minor was compared to Apatemon sp. Kruger, 1978, Apatemon graciliformis Szidat, 1928, and Cercaria 1 Richard, 1971.

Cercariae of the genus Cotylurus and Apatemon were easily identified from the number and distribution of acetabular papillae : $4 \mathrm{SI}+6 \mathrm{SII}$ in Cotylurus and $3 \mathrm{SI}+3 \mathrm{SII}$ in Apatemon. These characterisitics further enabled the identification of a cercaria belonging to the genus Diplostomum since all the carcariae studied in this genus have $3 \mathrm{SI}+6 \mathrm{SII}$.

* Faculté de Pharmacie, Laboratoire de Parasitologie - Illkirch, B. P. 10, F 67048 Strasbourg Cedex. Accepté le 8 février 1982 
Apatemon graciliformis is an exception. However we observed variations in the acetabular papillae in Cercaria 1, Apatemon graciliformis might then correspond to a stage in papillary evolution, but in the absence of chaetotaxic investigations for intraspecies variations in the number and distribution of acetabular papillae, we cannot rule out the hypothesis that the specis may be related to a subgenus or even a different genus. This however requires clarification through chaetotaxic analysis of the other species.

Deux furcocercaires appartenant à la famille des Strigéidés (tribu Cotylurini Dubois, 1936) ont été trouvées chez des Planorbidés récoltés dans des mares de la forêt du Rhin au Nord de Strasbourg.

La première, parasite de Segmentina nitida Müller ${ }^{1}$, est celle de Cotylurus brevis Dubois et Rausch, 1950 (= Cercaria helvetica XXXIV, Dubois).

La seconde, parasite de Gyraulus acronicus Ferussac, est celle d'Apatemon (Australapatemon) minor Yamaguti, $1933^{2}$.

La morphologie des cercaires et des stades larvaires ayant servi à la détermination sera brièvement rappelée puis la répartition des cils sera décrite chez les cercaires.

\section{Les stades larvaires de Cotylurus brevis Dubois et Rausch, chez Segmentina nitida}

\section{A - Rappel de leur morphologie}

Trois stades du cycle biologique de ce Trématode peuvent être observés chez le Mollusque : les sporocystes, les cercaires, les métacercaires.

Les sporocystes ( fig. 1 C) sont longs, ténus, à paroi mince et blancs. L'une des extrémités est plus large et renflée en ampoule. Les dimensions de six sporocystes varient de 1,9 à $2,3 \mathrm{~mm} / 0,08 \mathrm{~mm}(0,1$ à 0,4 à l'extrémité la plus large).

Les furcocercaires ( $f i g .1 A, B$ ) : Les dimensions de quatre cercaires fixées sont les suivantes : Corps 196-220/56-70 $\mu \mathrm{m}$. Tronc caudal : 182-196/30-43 $\mu \mathrm{m}$. Fourchons caudaux : 156-166/10-12 $\mu \mathrm{m}$. Organe adhésif : 42-45/32-35 $\mu \mathrm{m}$ Ventouse ventrale : 25-36/30-38 $\mu \mathrm{m}$, portant 4 rangées concentriques d'épines. La lèvre dorsale de l'orifice buccal est armée de deux rangées composées chacune d'une douzaine d'épines très courtes. Le pharynx de $12 \mu \mathrm{m}$ de diamètre est suivi d'un oesophage très court bifurquant immédiatement en deux coecums longs, atteignant l'extrémité postérieure du corps. Il y a deux paires de cellules glandulaires situées juste en avant de l'acetabulum. Le système escrêteur comprend 20 protonéphridies distribuées suivant la formule $2((2+2)+(2+2+(2))$; une commissure antérieure est visible juste en avant de la ventouse. Deux taches oculaires non pigmentées mais très réfringentes sont situées latéralement et en avant de l'acetabulum.

\footnotetext{
I. Les Mollusques ont été déterminés par H. Chevallier que nous remercions.

2. Nous remercions G. Dubois qui a bien voulu examiner nos dessins et nous conseiller pour déterminer les cercaires.
} 


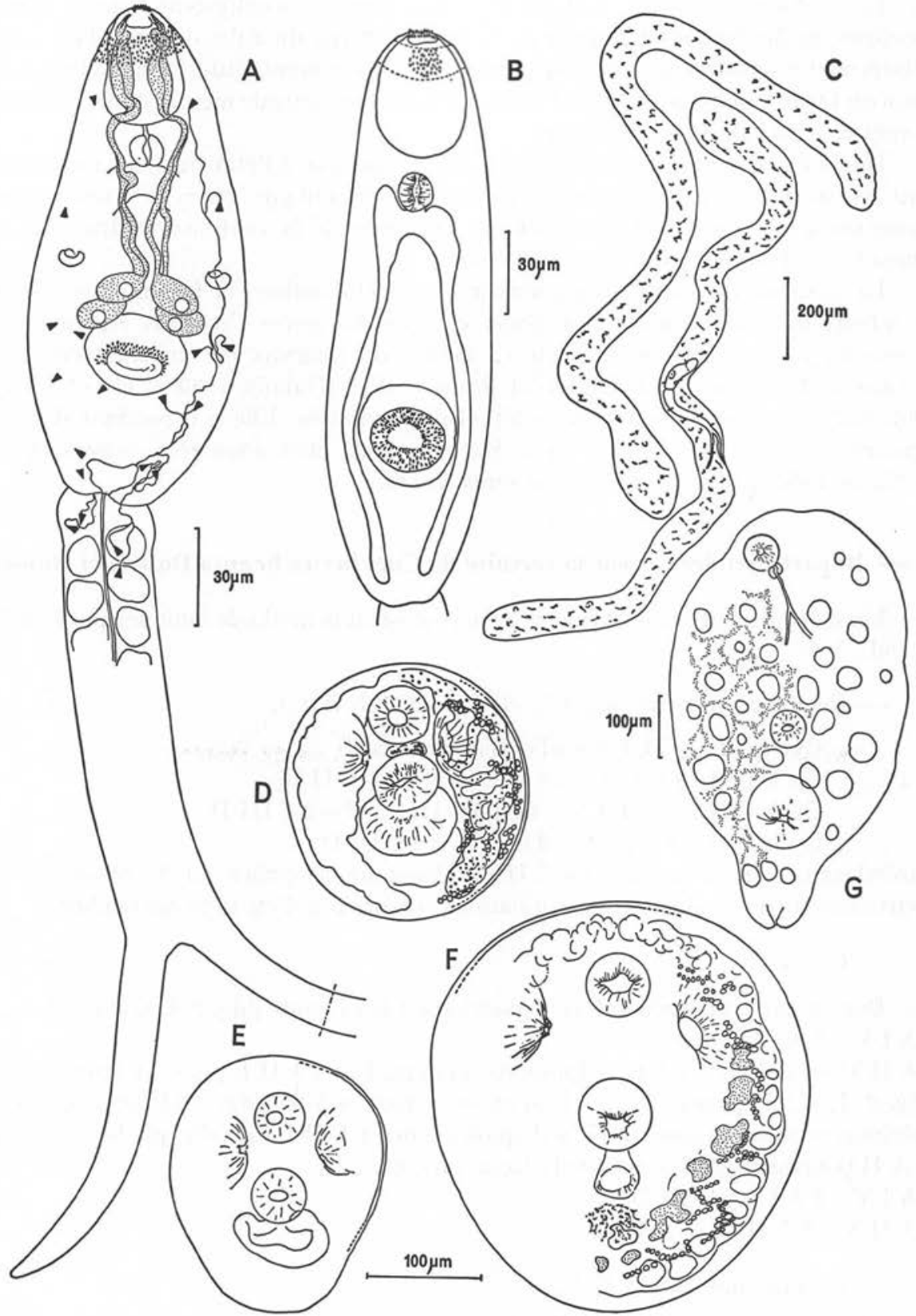

FIG. I. - Cotylurus brevis, stades larvaires. A : cercaire vivante. B : cercaire fixée, C : sporocystes, D, E, F : métacercaires enkystées, G : métacercaire dékystée. 
Les métacercaires (fig. $1 \mathrm{D}, E, F$ ) : Les cercaires s'enkystent dans la glande digestive du Mollusque et le long de la paroi externe du tube digestif. Les kystes aplatis sont circulaires à ovales ou piriformes. Ils mesurent 210-350/205-325 $\mu \mathrm{m}$. La paroi du kyste a une épaisseur de 4-5 $\mu \mathrm{m}$. La ventouse ventrale mesure 45-60/48-60 $\mu \mathrm{m}$ la ventouse buccale $45-60 / 50-68 \mu \mathrm{m}$.

Une métacercaire dékystée $(f i g .1 \mathrm{G})$ a été mesurée à l'état frais, ses dimensions sont les suivantes : longueur/largeur du corps : 720/440 $\mu \mathrm{m}$. Pharynx : 100/150 $\mu \mathrm{m}$. Diamètre de la ventouse buccale $60 \mu \mathrm{m}$. Diamètre de la ventouse ventrale $60 \mu \mathrm{m}$. Diamètre de l'organe tribocytique $125 \mu \mathrm{m}$.

La présence de 4 cellules glandulaires préacétabulaires, la formule du système excréteur, indiquent qu'il s'agit d'une cercaire du genre Cotylurus Szidat, 1928. L'oesophage court bifurquant juste en arrière du pharynx est un caractère de la cercaire de Cotylurus brevis Dubois et Rausch, 1950 (Dubois, 1938 p. 119 et 1968 p. 196). Cette cercaire a pour hôte habituel des Lymnées. Elle a cependant déjà été signalée chez des Planorbidés : par Mathias, 1925 chez Planorbis corneus et par Golikova 1960 également chez Segmentina nitida.

\section{B - Répartition des cils sur la cercaire de Cotylurus brevis Dubois et Rausch.}

Les cercaires ont été imprégnées à l'argent selon la méthode indiquée par Combes et coll., 1976.

- Région céphalique (fig. $2 \mathrm{G}, \mathrm{H}, \mathrm{I}$ et fig. $3 \mathrm{~A}, \mathrm{~B}, \mathrm{C}$ ).

Cycle C I : 1 C I V dans l'entonnoir buccal

Cycle C II : 1 C II V - 10 à 12 (C II L + C II D)

Cycle C III : 1 C III V -4 ou 5 C III L $-1+2$ C III D

Cycle C IV : 1 C IV V - 3 C IV L - 4 C IV D

Latéralement, au niveau du cycle C III, 3 plages argentophiles ont été observées qui pourraient correspondre à des terminaisons sensorielles d'un type particulier.

- Corps

Dans le cas le plus général, la disposition est la suivante ( $f i g .2 \mathrm{~A}, \mathrm{~B}$ et fig. $3 \mathrm{~A}$ ). $1 \mathrm{~A} I \mathrm{~V}-3 \mathrm{~A} I \mathrm{D}$

$1 \mathrm{~A}$ II V - 4 A II L-1 A II D (présente ou non). Les 4 A II L peuvent être groupées (fig. $2 \mathrm{~A}, \mathrm{G}$ ), alignées ( fig. $2 \mathrm{~F}$ ) ou en deux parties $3+1$ (fig. $2 \mathrm{I}$ ). Dans le cas du spécimen représenté figure $2 \mathrm{~F}$, la disposition des $4 \mathrm{~A}$ II L est telle que les cycles A I et A II pourraient être décrits de la façon suivante :

$1 \mathrm{~A}$ I V $-2 \mathrm{~A}$ I L $-2 \mathrm{~A}$ I D

1 A II V-2 A II L-1 A II D

- Acetabulum (fig. 2 A, E)

Il y a deux cercles de papilles. Le cercle interne SI a 4 papilles, parfois 5 . Le cercle externe SII en a toujours, sans exception 6. 


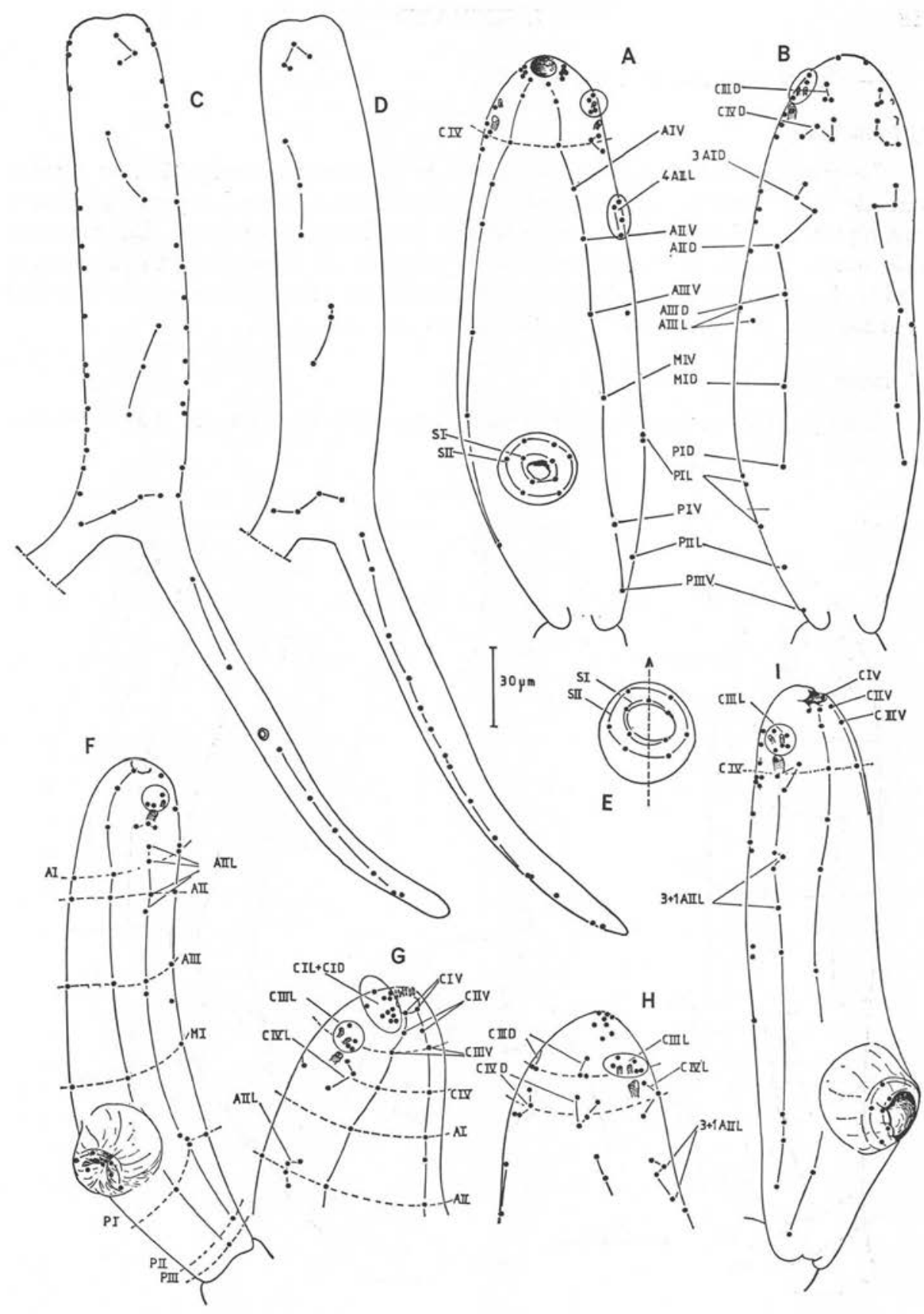

FIG. 2. - Cotylurus brevis, répartition des cils. A : corps, vue ventrale, B : corps, vue dorsale, $\mathrm{C}$ : queue, vue dorsale. $\mathrm{D}$ : queue, vue ventrale, $\mathrm{E}$ : acetabulum. $\mathrm{F}, \mathrm{I}$ : corps vue latéro-ventrale, $\mathrm{G}$ : région antérieure vue latéro-ventrale, $\mathrm{H}$ : région antérieure, vue latéro-dorsale. 
- Queue (fig. $2 C, D$ et fig. $3 D, E$ )

a) tronc caudal

Les papilles ventrales et dorsales n'ont pas une répartition régulière. Le nombre varie de 10 à 13 pour les ventrales et de 13 à 15 pour les dorsales. Les papilles latérales sont réparties en deux séries et sont généralement disposées par paires. Une première série occupe le cinquième antérieur de la longueur et comprend généralement 4 papilles (parfois 3 ou 5). La deuxième série occupe les $2 / 3$ postérieurs de la longueur et se compose de 12 papilles. Ce nombre peut varier de 11 à 13 .

\section{b) furcas}

Elles portent un nombre de papilles compris entre 25 et 28, soit 8 à 10 dorsales et 17 à 18 ventrales.

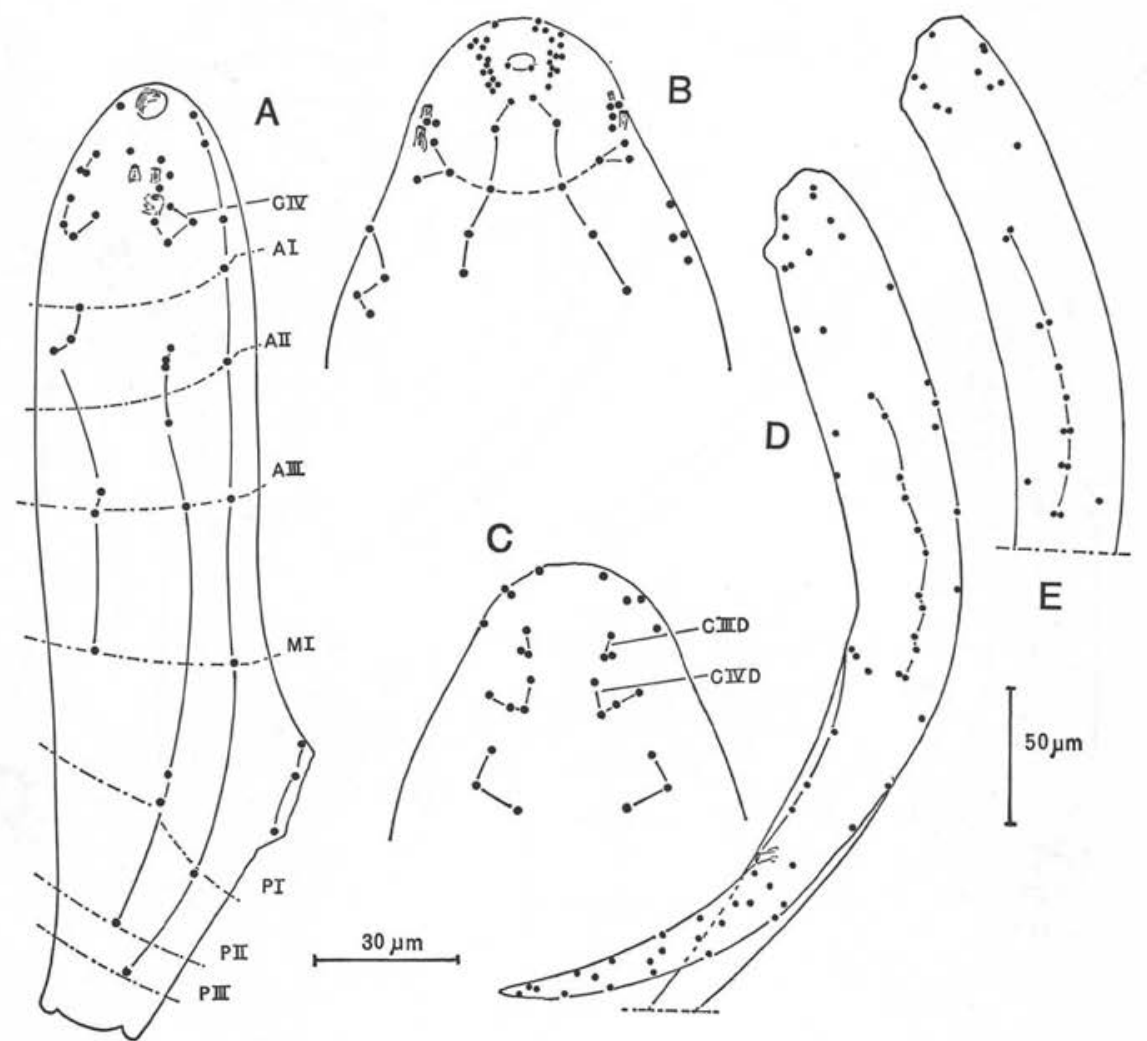

FIG. 3. - Cotylurus brevis, répartition des cils. A : corps vue latérale, $\mathrm{B}$ et $\mathrm{C}$ région antérieure vues ventrale et dorsale, D : queue vue latérale du côté droit, E : tronc caudal, vue latérale du côté gauche. 


\section{Les stades larvaires d'Apatemon (A.) minor chez Gyraulus acronicus}

\section{A - Rappel de leur morphologie.}

Les sporocystes ( $f$ ig. $4 \mathrm{~A}$ ) sont fins, transparents de longueur très variable. Pour trois d'entre eux les dimensions varient de $3,5-8,2 / 0,2-1 \mathrm{~mm}$.

Les furcocercaires ( $f \mathrm{~g} .4 \mathrm{~B}-\mathrm{C}$ ). Leurs dimensions sont les suivantes : Corps: 124-138/60-70 $\mu \mathrm{m}$. Tronc caudal : 140-160/38-42 $\mu \mathrm{m}$, contenant 16 " caudal bodies " très mobiles. Fourchons caudaux : 120-136/9-10 $\mu$ m. Organe adhésif 38/32 $\mu \mathrm{m}$.

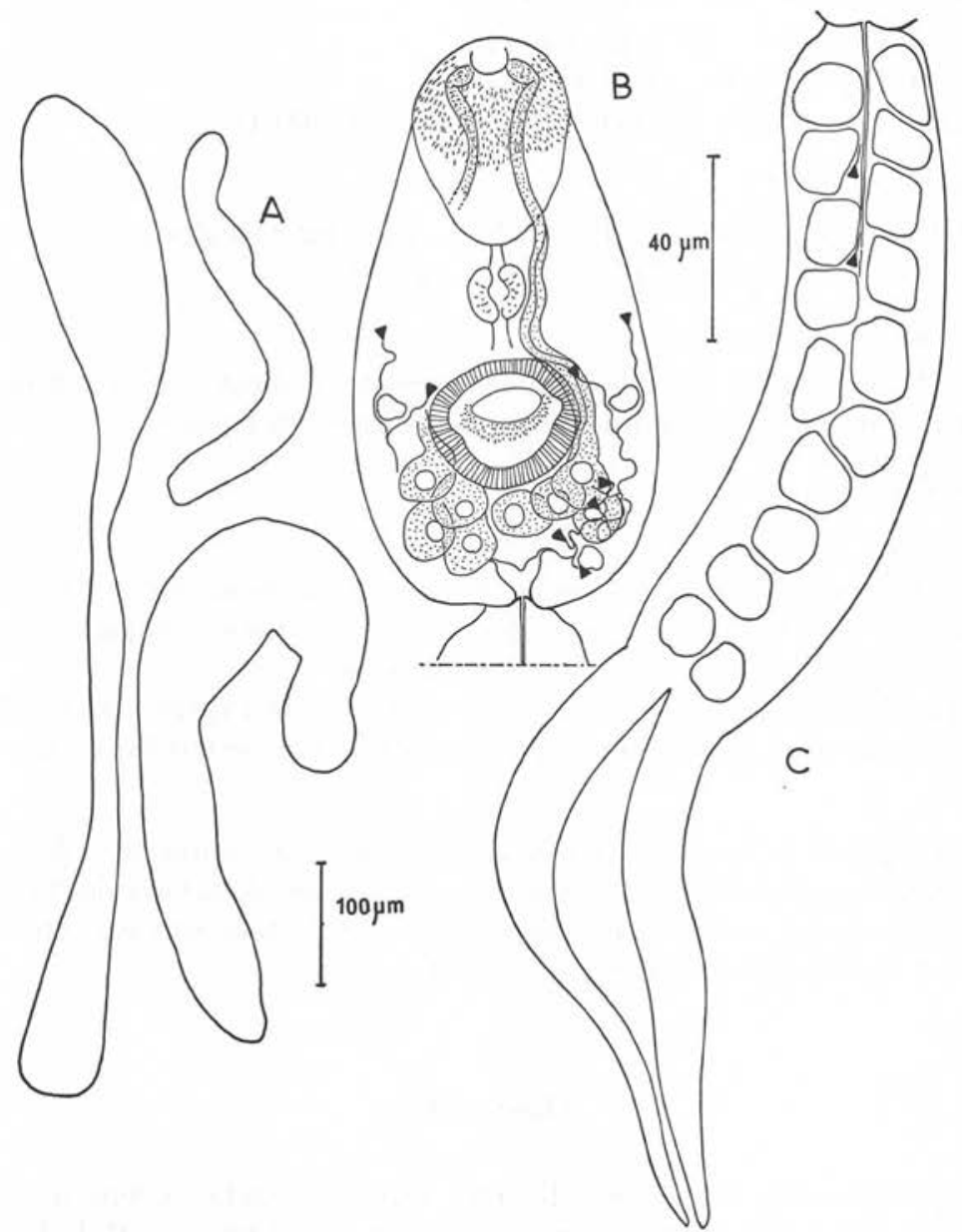

FIG. 4. - Apatemon (A.) minor, stades larvaires. A : sporocystes, B : corps de la cercaire, C : queue de la cercaire. 
Pharynx 12-15/11-12 $\mu \mathrm{m}$. Ventouse ventrale 32-33/34-36 $\mu \mathrm{m}$, armée de 4 rangées concentriques de petites épines. Les cellules glandulaires au nombre de 4 paires sont situées en arrière de l'acetabulum. Le système excréteur comprend 14 protonéphridies disposées suivant la formule $2[(1+1+(2+2+(1))]$.

Les caractères anatomiques (glandes de pénétration, formule excrétrice, disposition des "Caudal bodies ") et les dimensions, correspondent à ceux de la cercaire d'Apatemon (A.) minor Yamaguti, 1933.

\section{B - Répartition des cils sur la cercaire d'Apatemon (A.) minor Yamaguti.}

- Région céphalique (fig. $5 \mathrm{~A}$ à $\mathrm{D}$ et $\mathrm{F}$ )

Cycle C I : 1 C I V-8 C I L-2 C I D

Cycle C II : 1 C II V - 1 C II L

Cycle C III : $1+3$ (à 5) C III V - 8 C III L - 4 C III D

- Corps (fig. $5 B$ à $F$ )

$1 \mathrm{~A} \mathrm{I} \mathrm{V} \mathrm{(parfois} \mathrm{absente)} \mathrm{-} 1$ à $3 \mathrm{~A} \mathrm{I} \mathrm{L} \mathrm{-} \mathrm{A} \mathrm{I} \mathrm{D} \mathrm{(parfois} \mathrm{absente)}$

Pas d'A II V- 1 ou 2 A III L - 2,3 ou 4 P II L

- Acetabulum (fig. 5 B, E)

$3 \mathrm{SI}$ formant un triangle dont le sommet est dirigé vers l'arrière et $3 \mathrm{SII}$ formant un triangle dont le sommet est dirigé vers l'avant.

- Queue (fig. 5 G, H)

a) tronc caudal

Il y a des papilles ventrales, dorsales et latérales. Leur nombre est variable, 10 à 13 dorsales, 11 à 15 ventrales. Seul le groupe le plus antérieur apparaît constant et composé de 4 papilles dorsales et 3 papilles ventrales.

Les papilles latérales (de 10 à 12), sont disposées de l'avant vers l'arrière de la manière suivante : 1,1 , puis 5 paires réparties sur la moitié postérieure du tronc caudal. b) furcas

Il y a 6 paires de papilles sur chacune des furcas (6 ventrales, 6 dorsales). Les deux premières papilles dorsales et ventrales sont alignées dans l'axe des furcas, à leur naissance. Les quatre autres paires sont situées dans la zone comprise entre l'orifice excréteur et l'extrémité et sont régulièrement espacées.

\section{Discussion}

Quelques auteurs, Basch 1969, Kruger, 1978, ont étudié la répartition des cils chez des cercaires appartenant aux genres Cotylurus et Apatemon. P. F. Basch, 1969 indique très sommairement la position de quelques cils ventraux et dorsaux sur le 


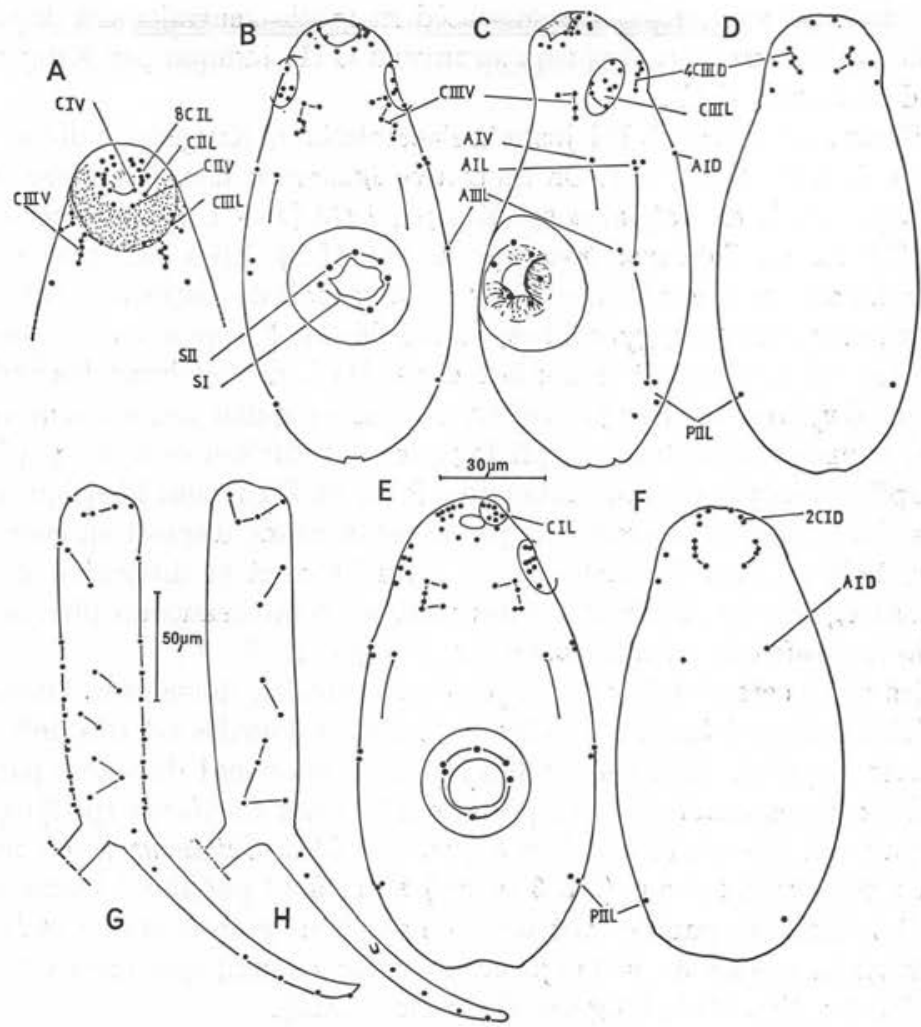

FIG. 5. - Apatemon (A.) minor, répartition des cils. A : apex de la cercaire, B : corps vue ventrale, C : vue latérale, D : corps vue dorsale, E et F : vues ventrale et dorsale d'un spécimen, $\mathrm{G}$ : queue vue ventrale, $\mathrm{H}$ : queue vue dorsale.

corps, sur le tronc caudal et les furcas de la cercaire de Cotylurus lutzi. La description est incomplète et ne peut être comparée à celle de Colyturus brevis. Un seul caractère, bien évident, est commun aux deux espèces : il s'agit des papilles acétabulaires S II au nombre de 6 , très schématiquement disposées suivant un hexagone régulier.

P. W. Kruger, 1978 décrit de façon très détaillée la chétotaxie d'une cercaire de Cotylurus sp. et d'une cercaire d' Apatemon sp.

Cotylurus sp. Kruger, 1978 a un certain nombre de points communs avec Cotylurus brevis, mais n'est pas identique. Le caractère commun le plus évident concerne les cycles acétabulaires S I + S II soit $4+6$ papilles ayant la même disposition chez les deux cercaires.

Un autre caractère est commun aux deux espèces. Il s'agit du nombre et de la succession des papilles céphaliques et antéacétabulaires ventrales. Le niveau C III L indiqué par Kruger correspondrait au niveau C IV que nous avons adopté pour 
Cotylurus brevis. En effet, 4 papilles se succèdent médio-ventralement depuis le bord antérieur de l'ouverture buccale jusqu'au niveau C III indiqué par Kruger. Il s'agit donc bien d'un niveau C IV.

Par ailleurs, du niveau C III jusqu'à l'acétabulum, Kruger a indiqué 4 papilles successives A I, A II, A III, M I. On les trouve également chez Cotylurus brevis.

Le groupe C II L de Cotylurus sp. Kruger, 1978 ( $7+1$ papilles) correspondrait au groupe C III L de Cotylurus brevis (4 ou 5 C III L). Elles sont plus nombreuses dans le premier cas car il semble que Kruger n'a pas fait de distinction entre les insertions ciliaires et les plages argyrophiles, au nombre de 3 , que nous signalons dans la description (et qui porteraient le nombre des C III L de Cotylurus brevis à 7 ou 8), soit parce que Cotylurus sp. Kruger, 1978 n'a que des papilles soit parce que ces plages ne sont pas apparues à l'auteur morphologiquement différentes d'une papille.

Les papilles ventrales postacétabulaires P I V et P I L sont identiques.

Les papilles dorsales ne semblent pas avoir la même disposition chez Cotylurus sp. Kruger, 1978 et chez Cotylurus brevis. Le nombre et la diposition des papilles caudales sont également différents. Elles sont au total beaucoup plus nombreuses chez Cotylurus brevis que chez Cotylurus sp. Kruger, 1978.

Chez les deux cercaires il y a des papilles ventrales, dorsales et latérales sur le tronc caudal. La disposition des papilles ventrales et dorsales est très différente chez l'une et l'autre espèces. Pour les papilles latérales, elles sont disposées par paires et occupent deux zones antérieures et postérieures. Chez Cotylurus sp. Kruger, 1978, indique 3 paires à l'avant et 5 paires à l'arrière. Chez Cotylurus brevis nous avons trouvé 4 à 5 papilles à l'avant (2 à 3 paires) et 11 à 13 papilles à l'arrière (environ 6 paires). Ces dernières papilles ont une étendue plus grande chez Cotylurus brevis (elles occupent la moitié de la longueur du tronc caudal) que chez Cotylurus sp., Kruger, 1978, (un tiers de la longueur du tronc caudal).

Pour résumer, Cotylurus brevis et Cotylurus sp., Kruger, 1978, ont en commun quelques caractères et diffèrent essentiellement par le nombre et la disposition des papilles dorsales du corps et les papilles caudales.

La cercaire d'Apatemon sp., décrite et représentée par Kruger, 1978, est proche d'Apatemon (A.) minor. Comme dans le cas précédemment discuté, le caractère commun le plus marquant porte sur les cycles de papilles acétabulaires S I + S II, soit $3+3$ papilles dont la disposition est la même chez les deux cercaires.

Pour les cycles céphaliques, il semble y avoir une certaine similitude entre les cycles C II (V et L), C III (V et L) d'Apatemon sp., Kruger, 1978, et le cycle C III d'Apatemon (A.) minor. Il semblerait en effet, que les deux cycles, parfaitement individualisés chez Apatemon sp., Kruger, 1978, se sont nettement rapprochés l'un de l'autre voir même téléscopés chez Apatemon (A.) minor entraînant la disparition d'un certain nombre de papilles, en particulier celle de grand diamètre représentée par Kruger dans le groupe C III V. Chez Apatemon sp., Kruger, 1978, l'ensemble C II V - C II L, C III V - C III L, correspond à 17 papilles. Les C III V et C III L chez Apatemon (A.) minor se composent de 14 papilles.

Les papilles céphaliques dorsales représentées figure 16 par Kruger sont identiques à celles de la figure $5 \mathrm{D}$ du présent travail. 
Pour les papilles ventrales et dorsales sur le corps des deux cercaires, les A I V, A I L, les M I L d'Apatemon sp., Kruger, 1978, les A III L d'Apatemon (A.) minor, et le cycle P II sont identiques. Chez Apatemon sp., Kruger, 1978, il semble que la P II V soit nettement séparée des P II L, alors qu'elles ne forment qu'un seul groupe chez Apatemon (A.) minor.

Dorsalement le corps des deux cercaires est différent. Il y a une M I D chez Apatemon sp., Kruger, 1978, et une A I D (très nettement à l'avant du niveau M I) chez Apatemon (A.) minor.

En ce qui concerne la queue, chez les deux cercaires, des papilles ventrales, dorsales et latérales sont présentes.

Ventralement et dorsalement, chez les deux cercaires, il y a 3 papilles du côté proximal du tronc caudal. Les autres papilles ont une réparttion différente et sont plus nombreuses chez Apatemon (A.) minor que chez Apatemon sp.

Chez Apatemon (A.) minor, latéralement, l'extrémité proximale du tronc caudale porte deux papilles largement écartées et la moitié distale porte 4 à 5 paires de papilles.

Chez Apatemon sp., Kruger ne signale pas la présence de papilles latérales dans la partie proximale du tronc caudal et il n'y en aurait que 3 paires dans la partie distale.

La face ventrale des furcas est identique chez ces deux Apatemon.

En résumé, les deux cercaires Apatemon sp., Kruger, 1978 et Apatemon (A.) minor sont très proches. La première pourrait appartenir à un sous genre différent, en raison du caractère très particulier des cycles céphaliques qui apparaissent bien individualisés chez cette cercaire alors qu'ils se confondent chez Apatemon (A.) minor.

Apatemon (A.) minor est également très proche de Cercaria 1 trouvée en grande Brière chez Planorbis carinatus (Richard, 1971).

Pour les papilles situées sur le corps et sur la queue, leur nombre et leur disposition sont proches chez les deux cercaires. Les variations d'un échantillon à l'autre qui pourraient être observées chez l'une, le sont également chez l'autre (par exemple l'instabilité des A II V, le nombre des papilles A II L, P II L).

Toutefois, chez la cercaire d'Apatemon (A.) minor, au niveau céphalique, le groupe C III L est composé d'un nombre plus faible de papilles, 8, au lieu de 10 à 13 chez Cercaria 1 (nombre qui tend à correspondre à celui indiqué par Kruger chez Apatemon sp.) Chez Cercaria 1, les papilles acétabulaires ont dans de nombreux cas la disposition classique rencontrée chez Apatemon (A.) minor et chez Apatemon sp., c'est-à-dire S I + S II $=3+3$. Cependant, des variations numériques sont observées chez cette cercaire (Richard, 1971, pl. 11) alors qu'elles le sont rarement chez Apatemon (A.) minor.

Apatemon (A.) minor a des caractères communs avec Apatemon graciliformis Szidat, $1928^{1}$ : les A I V, A I L, A I D, A III V, A III L, les C I D d'Apatemon graciliformis et les C III D d'Apatemon (A.) minor, les papilles de la face ventrale des

I. C. Bayssade-Dufour nous a communiqué les dessins de la cercaire d'Apatemon graciliformis dont l'étude est en cours. Nous l'en remercions. 
furcas depuis l'orifice excréteur jusqu'à l'extrémité. Les autres papilles (cycles céphaliques ventraux, cycles postacétabulaires P I et P III, tronc caudal, acetabulum) sont différents chez ces deux espèces.

L'ensemble des cercaires de Strigeidae abordées dans ce travail Cotylurus brevis Dubois et Rausch, 1950, Cotylurus Lutzi Basch, 1969, Cotylurus sp., Kruger, 1978, Apatemon (A.) minor Yamaguti, 1933, Apatemon sp., Kruger, 1978, Cercaria 1 Richard, 1971, Apatemon graciliformis Szidat, 1928, peut, d'après la chétotaxie être partagé en deux groupes :

1 - Les cercaires appartenant au genre Cotylurus : L'acetabulum porte 4 (ou 5) +6 papilles. Les cils latéraux sont présents dans la partie proximale du tronc caudal (2 à 3 paires) et dans la partie distale du tronc caudal (5 à 6 paires). La face dorsale des furcas porte au minimum 6 papilles dans la zone s'étendant de l'orifice excréteur à l'extrémité.

2 - Les cercaires appartenant au genre Apatemon : Le nombre de papilles acétabulaires peut varier d'un individu à l'autre, mais il semble tendre vers un type stable à $3 \mathrm{~S} \mathrm{I}+3 \mathrm{~S}$ II. Les cils latéraux sont absents ou peu nombreux dans la partie proximale du tronc caudal ( 0 à 2 ou 3 cils) et ils sont présents dans la partie distale du tronc caudal (3 à 4 paires). La face dorsale des furcas porte 4 papilles dans la zone s'étendant de l'orifice excréteur à l'extrémité.

Le nombre et la disposition des papilles acétabulaires apparaissent comme un caractère des genres Cotylurus et Apatemon. Le cas d'Apatemon graciliformis semble particulier : il pourrait correspondre, dans un genre où les espèces sont instables, à une des étapes finales de la réduction de l'acetabulum et de son système sensoriel, puisqu'il n'y a plus que $1 \mathrm{~S} \mathrm{I}+2 \mathrm{~S}$ II. Il pourrait aussi s'agir d'un genre différent. En effet, chez les Strigeidae, dans le genre Diplostomum où la chétotaxie d'un certain nombre de cercaires est connue, le nombre et la disposition des papilles acétabulaires sont également constants. D. coniferum cf. Richard, 1971, D. gobiorum cf. Shigin, 1960, D. indistinctum cf. Shigin, 1968, D. mergi cf. Shigin, 1973, Cercaria petromyzi fluviatilis cf. Sweeting, 1976, Tylodelphys sp., cf. Kruger, 1978, ont $3+6$ papilles sur la ventouse ventrale.

En ce qui concerne les papilles latérales portées par le tronc caudal dans la zone proximale, elles sont présentes aussi bien chez Cotylurus sp. que chez Cotylurus brevis. Il est probable qu'elles sont présentes chez toutes les cercaires appartenant à ce genre dans la famille des Strigeidae. Elles sont également présentes dans le genre Diplostomum qui appartient à une famille différente, celle des Diplostomatidae. On ne peut donc, comme l'indique Bayssade-Dufour (1979) fonder la distinction entre les familles de Strigeoïdea sur le mode de répartition et la présence ou l'absence des cils proximaux et distaux sur le tronc caudal.

La disposition des papilles acétabulaires chez Cotylurus brevis permet une remarque de caractère évolutif. Il est indiqué dans la description que les cercaires ont $4 \mathrm{~S} \mathrm{I}+6 \mathrm{~S} \mathrm{II} \mathrm{à} \mathrm{l'exception} \mathrm{d'un} \mathrm{nombre} \mathrm{limité} \mathrm{qui} \mathrm{en} \mathrm{a} 5+6$. Dans ce dernier cas, il y a deux dispositions possibles des papilles S I, en fonction de la position de la cinquième papille. Celle-ci est en effet toujours dans le plan sagittal, soit à l'avant 
(fig. 2 E), soit à l'arrière (fig. 2I). Les 4 S I que l'on rencontre dans le genre Cotylurus, pourraient donc dériver d'un cycle S I à 6 papilles (disposées suivant un hexagone régulier), par disparition des papilles situées sur la diagonale qui est dans le plan sagittal.

\section{BIBLIOGRAPHIE}

BAsch P. F. : Cotylurus lutzi sp. n. Trematoda Strigeidae and its life cycle. J. Parasitol., 1969, 55, 527-539.

BAYSSADE-DUfour C. : L'appareil sensoriel des cercaires et la systématique des Trématodes digénétiques. Mem. Mus. Nat. Hist. Nat., 1979, 113 , série A, Zool., I-81.

Combes Cl, BAySSADE-Dufour C., CASSONE J. : Sur l'imprégnation et le montage des cercaires pour l'étude chétotaxique. Ann. Parasitol. Hum. Comp., 1976, 51, 399-40o.

Dubors G. : Monographie des Strigéida (Trematoda). Mém. Soc. Neuchâtel. Sci. Nat., I938, 6, I-535.

Dubors G. : Synopsis des Strigeidae et des Diplostomatidae (Trematoda). Mém. Soc. Neuchâtel. Sci. Nat. (I er fasc.), I960, I-258.

Golikova M. N. : Ecological and parasitological investigation of the biocenosis of some lakes in the kaliningrad region IV, on the Trematode fauna of the Invertebrates. Vestnik Leningrad Univ., 1960, 21, s. biol., 80-94.

KRUGER P. W. : Chaetotaxic studies on some local furcocercous cercariae and their sporocysts. B. Med. Sci., Thesis, University of Queensland, 1978, 54 p. polycopiées.

Mathias P. : Recherches expérimentales sur le cycle évolutif de quelques Trématodes. Bull. Biol. France et Belgique, 59 , 1925 , I-124.

Richard J. : La chétotaxie des cercaires. Valeur systématique et phylétique. Mém. Mus. Nat. Hist. Nat., 1971, 67, série A, Zool., I-179.

Shigrn A. A. : Contribution à la connaissance du cycle de développement et de la morphologie de la cercaire de Diplostomum indistinctum (Trématoda : Diplostomatidae). Trud. Gel'mint. Lab. Akad. Nauk. SSSR, Moscou, I968, 19, 208-227 (en russe).

SHigrn A. A. : Sur le cycle de développement et la validité spécifique de Diplostomum gobiorum Shigin, 1965 (Trematoda : Diplostomatidae). Trud. Gel'mint. Lab. Akad. Nauk. SSSR Moscou, 1969, 20, 176-190 (en russe).

ShigrN A. A. : L'appareil sensoriel des cercaires du genre Diplostomum (Trematoda : Diplostomatidae) et son importance taxonomique. Trud. Gel'mint. Lab. Akad. Nauk. SSSR, Moscou, 1973, 23, 186-195 (en russe).

SwEETING R. : An experimental demonstration of the life cycle of a Diplostomulum from Lampreta fluviatilis Linneaeus, 1758. Z. Parasitkde, 1978, 49, 233-242. 